\title{
ISAKOS celebrates 20 years of education, fellowship and outreach!
}

\author{
Philippe N. Neyret ${ }^{1} \cdot$ Marc R. Safran $^{2} \cdot$ Willem van der Merwe ${ }^{3} \cdot$ Masahiro Kurosaka $^{4}$
}

Published online: 19 September 2015

(C) European Society of Sports Traumatology, Knee Surgery, Arthroscopy (ESSKA) 2015

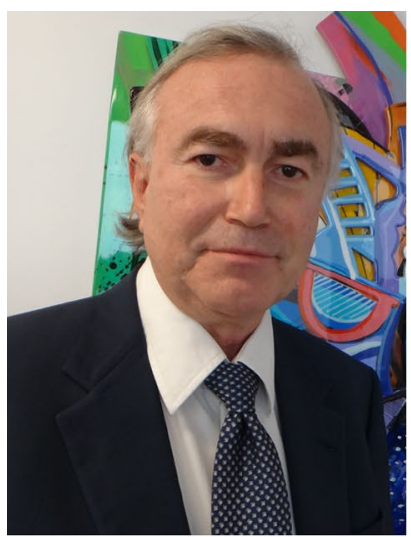

Philippe N. Neyret

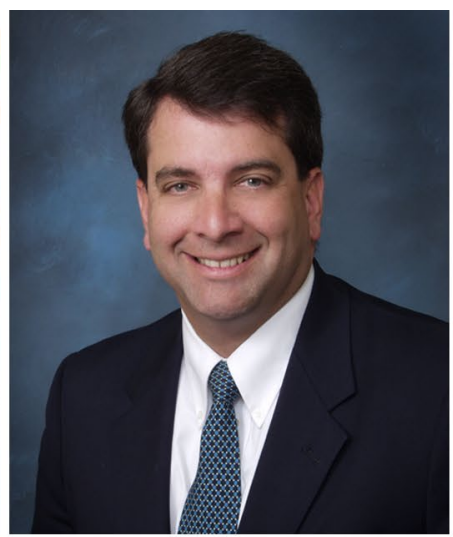

Marc R. Safran

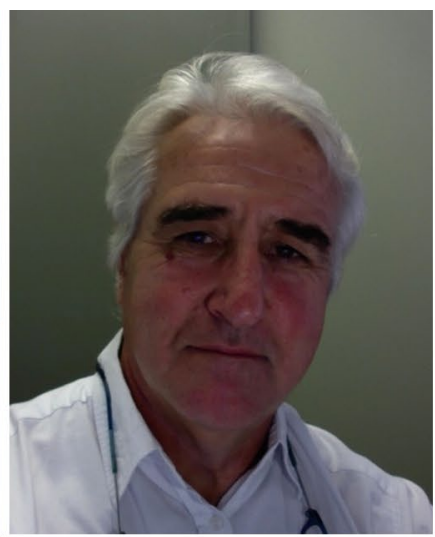

Willem van der Merwe

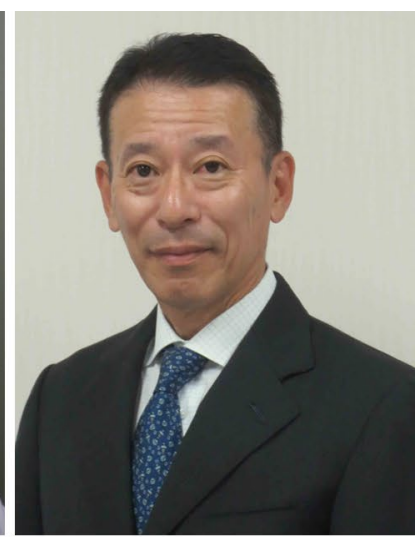

Masahiro Kurosaka
At 20 years old, ISAKOS is recognised as a leading worldwide organisation. ISAKOS was formed by the merger of the International Arthroscopy Association (IAA President H. Eikelaar) and the International Society of the Knee (ISK

Philippe N. Neyret

philippe.neyret@chu-lyon.fr

Marc R. Safran

msafran@stanford.edu

Willem van der Merwe

willem@ssoc.co.za

Masahiro Kurosaka

kurosaka@med.kobe-u.ac.jp

1 Hospices Civils de Lyon, Lyon 1 University, Lyon, France

2 Stanford University, Stanford, CA, USA

3 Sports Science Institute of South Africa, University of Cape Town, Cape Town, South Africa

4 Department of Orthopaedic Surgery, Graduate School of Medicine, Kobe University, Kobe, Japan
President R. Marti) in 1995 at their combined congress in Hong Kong. Today, ISAKOS is known worldwide as a premier organisation in the fields of arthroscopy, knee surgery and orthopaedic sports medicine.

Our mission has defined our history and continues to shape our future-ISAKOS is an international society of surgeons established to advance the worldwide exchange and dissemination of education, research, and patient care in arthroscopy, knee surgery and orthopaedic sports medicine.

ISAKOS strives to maintain diversity and perspective, as we serve our more than 3000 dedicated members, from 95 different countries. ISAKOS works with regional and national societies who share similar goals, providing a larger arena where these organisations can combine their strengths in an international forum. ISAKOS works in close collaboration with our partner societies ESSKA, AANA, AOSSM, APKASS and SLARD to fulfil our common mission of education. 
Membership growth has been rapid as a result of the many initiatives of the society, more than doubling in the last 10 years! Membership benefits include subscription to Arthroscopy: The Journal of Arthroscopic and Related Surgery, access to the ISAKOS Global Link-the ISAKOS educational portal, subscription to the ISAKOS Newsletter, opportunity to serve on committees, optional subscription to KSSTA, and support of meetings and educational experiences worldwide.

The ISAKOS Biennial Congress continues to be a hallmark event of the society. ISAKOS has held 10 incredibly successful ISAKOS Congresses around the world including Buenos Aires in 1997 (President P. Fowler); Washington, DC, in 1999 (President G. Poehling); Montreux in 2001 (President R. Jakob); Auckland in 2003 (President B. Tietjens); Hollywood, Florida, in 2005 (President P. Renstrom); Florence in 2007 (President J. Bergfeld); Osaka in 2009 (President P. Aglietti); Rio de Janeiro in 2011 (President F. Fu); Toronto in 2013 (President M. Cohen); and most recently Lyon in 2015 (President M. Kurosaka). The 2015 Congress had more than 4000 registered participants, 550 presenters, over 600 electronic posters, and 25 live surgical demonstrations!

ISAKOS would like to thank the international orthopaedic and sports medicine community for their supportconnecting people is part of the mission of our worldwide organisation! ISAKOS' collaborative efforts have included:

- Collaboration with partner societies, such as ESSKA, for regional education courses.

- Collaboration on ISAKOS Global Link with partner societies and their journals.

- Committee activities with participation of multiple regions on publications, research and online courses.

ISAKOS has some unique challenges, operating in a world that is ever expanding and shrinking with equal measure. While our online education and outreach continues to expand, we also encounter challenges with international regulations and working in a diverse global market. Language remains a distinct barrier to truly international education, but ISAKOS seeks to challenge this with our 'Teach the Teacher' initiatives. Teach the Teacher brings education to designated surgeons, with the hope that they will in turn take what they have learned and disseminate that information to their colleagues in an applicable and understandable way.

ISAKOS also continues to encourage research, not only with multicenter studies but 'multi-continental' studies. Some multi-continental studies were recently initiated, supported by ISAKOS. ISAKOS has also initiated a Young Investigator Research Mentoring Programme in which a young surgeon from a developing or underserved market is paired with a more experienced surgeon or researcher to complete a research project under their careful mentoring.

To enhance international education and improve patient care, ISAKOS has developed an international journal to meet the needs and expectations of our membership and the unique mission of this worldwide organisation. The spirit of Journal of ISAKOS is not to compete with the other excellent journals that already exist and are well established, but to offer a worldwide approach and to cover all the fields of the mission of ISAKOS and its specialties. Professor C. Niek van Dijk, the first Editor-in-Chief of JISAKOS and former President of ESSKA, will lead this very ambitious and exciting ISAKOS initiative.

ISAKOS is a society where surgeons from all around the world with different conditions, different ages, different knowledge and different cultures can meet and sharewe seek to be a platform for exchange in the international orthopaedic and sports medicine community. We look forward to your continued partnership with ISAKOS, or welcoming you to our international family if you have not experienced the fraternity that is ISAKOS! 